\title{
Tratamento cirúrgico da embolia pulmonar maciça aguda
}

\author{
Camilo ABDULMASSIH NETO*, Marcos A. O. BARBOSA**, Leopoldo S. PIEGAS ${ }^{*}$, Paulo CHACCUR*, \\ Jarbas J. DINKHUYSEN ${ }^{\star}$, Pedro R. SALERNO ${ }^{\star \star}{ }^{\star \prime}$ Antoninho S. ARNONI ${ }^{\star \star \star}$, Paulo P. PAULISTA ${ }^{\star \star \star}$, \\ Luiz Carlos Bento de SOUZA*, Adib D. JATENE**
}

RBCCV 44205-207

\begin{abstract}
ABDULMASSIH NETO, C.; BARBOSA, M. A. O.; PIEGAS, L. S.; CHACCUR, P.; DINKHUYSEN, J, J.; SALERNO, P. R.; ARNONI, A. S.; PAULISTA, P. P.; SOUZA, L. C. B.; JATENE, A. D. - Tratamento cirúrgico da embolia pulmonar maciça aguda. Rev. Bras. Cir. Cardiovasc., 8(2):125-129, 1993.
\end{abstract}

RESUMO: A embolia pulmonar maciça é uma afecção que exige diagnóstico e tratamento precoces, com a finalidade de reduzir a morbi-mortalidade, neste grave grupo de pacientes. No período compreendido entre janeiro de 1984 e dezembro de 1992, foram submetidos a tromboembolectomia por cirurgia 8 pacientes, acometidos de embolia pulmonar maciça. A idade variou de 36 a 70 anos com média de 56,6 anos, sendo 6 do sexo masculino. Destes, $6(75 \%)$ tinham antecedentes cirúrgicos recentes: revascularização do miocárdio em 3, lipoaspiração abdominal em 2 e hemorroidectomia em 1. Todos apresentavam níveis importantes de hipóxia, com paO $\mathrm{P}_{2}$ arterial, média de $55 \mathrm{~mm} / \mathrm{Hg}$. O diagnóstico foi confirmado em $6(75 \%)$ por angiografia pulmonar, com comprometimento arterial pulmonar superior a $70 \%$, e por cirurgia em 2 . 0 tratamento instituído foi a tromboembolectomia com circulação extracorpórea. O tempo transcorrido entre o início dos sintomas e a realização da operação $(T)$ variou de 2 horas a 72 horas, com média de 18,5 horas. $\mathrm{Na}$ evolução hospitalar, $4(50 \%)$ faleceram, sendo que 2 haviam apresentado parada cardíaca prévia. As causas de óbito foram: síndrome de angústia respiratória do adulto, 1; infecçāo pulmonar, 1; coma neurológico, 1 ; insuficiência miocárdica, 1 . Os 4 sobreviventes que tiveram boa evolução apresentaram T médio de 3,5 horas, bem inferiores aos que faleceram, cujo T médio foi de 33,5 . As cintilografias feitas entre $\circ 2^{2}$ e $\circ 7^{\circ}$ mês de pós-operatório mostravam apenas discretas alterações na perfusão pulmonar. Na evoluçāo tardia de 31 a 84 meses, 3 pacientes estão assintomáticos e 1 apresenta dispnéia aos grandes esforços. Baseados neste pequeno grupo podemos inferir:

1) a parada cardíaca prévia foi causa de mau prognóstico;

2) a precocidade de diagnóstico e tratamento foi fator preditivo de bom prognóstico;

3) a alta mortalidade justifica-se pela gravidade dos pacientes;

4) os pacientes sobreviventes apresentam boa evolução a longo prazo.

DESCRITORES: embolia pulmonar, cirurgia.

\section{INTRODUÇÃO}

Embora TRENDELENBURG 18 tenha sido o primeiro a sugerir a embolectomia pulmonar, no início de 1908 , seu discípulo KIRSCHNER ${ }^{12}$ realizou esta operação em 1924, com completa recuperação do paciente.
Nos anos seguintes, muitos tentaram duplicar este heróico e inusitado feito cirúrgico, mas sem sucesso.

Até 1961 , somente 23 citações de sobrevida a longo termo, puderam ser encontradas na literatura médica mundial.

Trabalho realizado no Instituto Dante Pazzanese de Cardiologia e no Hospital do Coração da Associação do Sanatório Sírio. São Paulo, SP, Brasil. Apresentado ao $20^{2}$ Congresso Nacional de Cirurgia Cardiaca. Maceió, AL, 2 e 3 de abril, 1993.

- Do Instituto Dante Pazzanese de Cardiologia e do Hospital do Coração.

* Do Hospital do Coração.

-.. Do Instituto Dante Pazzanese de Cardiologia.

Endereço para separatas: Camilo Abdulmassih Neto. Rua Desembargador Elizeu Guilherme, 123. Centro Cirúrgico. 04004-030 Paraíso, São Paulo, SP, Brasil. 
ABDULMASSIH NETO, C.; BARBOSA, M. A. O.; PIEGAS, L. S.; CHACCUR, P.; DINKHUYSEN, J, J.; SALERNO, P. R.; ARNONI, A. S.; PAULISTA, P.P.; SOUZA, L. C. B.; JATENE, A. D. - Tratamento cirúrgico da embolia pulmonar maciça aguda. Rev. Bras. Cir. Cardiovasc.,8(2):125-129, 1993.

Embolectomia pulmonar com sucesso, usando desvio cardiopulmonar foi relatado, em 1961, por COOLEY ot alii ${ }^{4}$.

Desde essa época, numerosos sucessos similares têm sido relatados e tornado aparente que a circulação extracorpórea (CEC) permite reanimar o paciente moribundo com embolia pulmonar maciça (EPM), e, o mais importante, propicia tempo suficiente para a remoção do coágulo da árvore arterial pulmonar.

\section{CASUÍSTICA E MÉTODOS}

No período de janeiro de 1984 a dezembro de 1992 , foram submetidos a tromboembolectomia por cirurgia 8 pacientes, acometidos de embolia pulmonar maciça. A idade variou de 36 a 70 anos, com média de 56,6 anos, sendo 6 do sexo masculino. Destes 8 pacientes, $6(75 \%)$ tinham antecedentes cirúrgicos: revascularização do miocárdio em $3(50 \%)$, lipoaspiração abdominal em 2 (33,3\%) e hemorroidectomia em 1 (16,6\%). Todos apresentavam níveis importantes de hipóxia com $\mathrm{paO}_{2}$ arterial médio de $55 \mathrm{~mm} / \mathrm{Hg}$. O diagnóstico foi confirmado em $6(75 \%)$ por angiografia pulmonar com comprometimento arterial pulmonar superior a $70 \%$ e por cirurgia em $2(25 \%)$. Um destes pacientes apresentou falha precoce com tratamento tromboembolítico.

O tratamento instituído foi a tromboembolectomia com auxílio da circulação extracorpórea. Através de esternotomia mediana, seguida de canulação da aorta e de ambas as cavas, instituiu-se a circulação extracorpórea normotérmica. Sem o pinçamento da aorta, o tronco pulmonar foi aberto, com ampliação para a artéria pulmonar. No último paciente da série, houve necessidade de dissecar a direita e abrí-la. Foi, então, realizada a retirada dos trombos do tronco e das artérias pulmonares, com o auxílio de pinça sacatrombo.

Como manobra auxiliar, foram abertas as pleuras e, com massagem do parênquima pulmonar, trombos que por ventura pudessem estar mais periféricos foram retirados. Em seguida, arteriorrafia pulmonar, seguida de saída da circulação extracorpórea. Um aspirador comum é usado para auxiliar na remoção dos coágulos distais. $O$ átrio e o ventrículo direitos são explorados e a veia cava inferior é levada com o próprio sangue, para eliminar coágulos residuais.

O tempo transcorrido entre o início dos sintomas e a realização da operação variou de 2 a 72 horas, com média de 18,5 horas.

\section{RESULTADOS}

$\mathrm{Na}$ evolução hospitalar, $4(50 \%)$ pacientes faleceram, sendo que 2 haviam apresentado parada cardíaca prévia ao tratamento cirúrgico. As causas de óbito foram: síndrome de angústia respiratória do adulto, 1 paciente; infecção pulmonar, 1; coma neurológico, 1 e insuficiência miocárdica 1.

Os 4 sobreviventes que tiveram boa evolução apresentaram tempo médio precedendo a operação de 3,5 horas, bem inferiores aos que faleceram, cujo tempo médio foi de 33,5 horas.

As cintilografias feitas entre $\circ 2^{\circ}$ e $\circ 7^{\circ}$ mês de pós-operatório mostraram apenas discretas alterações na perfusão pulmonar.

$\mathrm{Na}$ evolução tardia de 31 a 84 meses, 3 pacientes estão assintomáticos e 1 apresenta dispnéia aos grandes esforços.

\section{COMENTÁRIOS}

Embolismo pulmonar é uma entidade clínica comum; $50 \%$ em pacientes hospitalizados, freqüentemente como uma complicação após cirurgia 7 ; $75 \%$ dos nossos pacientes tinham operação prévia.

Entre pacientes submetidos a cirurgia geral, a incidência de embolia pulmonar fatal é de $0,8 \%$ a $1,0 \%{ }^{7}$. Embolismo pulmonar fulminante, resultando em hipóxia e falência ventricular direita, tem uma alta taxa de mortalidade.

$\mathrm{Na}$ literatura, temos encontrado que a taxa de mortalidade por obstrução de $50 \%$ da vasculatura pulmonar chega a $50 \%$ e sobe a $70 \%$ se o paciente requer terapêutica vasopressora. Se a deterioração clínica continua, a taxa de mortalidade aproxima-se a $100 \% 11$.

Muitos pacientes morrem sem ter sido referidos para uma unidade de cirurgia cardíaca ${ }^{4}$.

Uma das objeções para indicação de embolectomia pulmonar tem sido baseada no fato de que algumas operações foram realizadas, e continuam sendo, em pacientes que não têm diagnóstico comprovado de embolismo pulmonar maciço ${ }^{2}$.

Outros têm propalado que o paciente moribundo com EPM precisa permanecer vivo por um minimo de uma hora pós angiografia, para que a embolectomia possa ser efetuada 2 .

Este argumento é antiquado e cai com os avanços tecnológicos, incluindo equipamento de CEC portátil e uma equipe organizada e hábil. Nos nossos pacientes, o tempo entre o diagnóstico $\theta$ a 
ABDULMASSIH NETO, C.; BARBOSA, M. A. O.; PIEGAS, L. S.; CHACCUR, P.; DINKHUYSEN, J, J.; SALERNO, P. R.; ARNONI, A. S.; PAULISTA, P.P.; SOUZA, L. C. B.; JATENE, A. D. - Tratamento cirúrgico da embolia pulmonar maciça aguda. Rev. Bras. Cir. Cardiovasc.,8(2):125-129, 1993.

embolectomia é o principal fator preditivo de bom resultado.

Alguns têm chegado ao extremo e têm dito que a embolectomia pulmonar nunca é indicada; não obstante, um número substancial de pacientes continua morrendo a cada ano.

O diagnóstico é usualmente sugerido pela história de colapso circulatório agudo, com progressiva hipóxia e um aumento na necessidade de catecolaminas ${ }^{14}$. Isto é suportado pelos raios $X$ de tórax, eletrocardiograma (ECG) e cateter de Swan-Ganz. Evidência direta de embolismo pulmonar é provada por cintilografia, ventilação-perfusāo ou angiografia pulmonar, esta última sendo o método mais acurado e recomendado por vários autores ${ }^{19}$.

CLARKE ${ }^{2}$ afirma que a angiografia e a cintilografia ventilação-perfusão não são mandatórias como procedimento diagnóstico, podendo causar um inaceitável atraso e a morte ocorrer em algum momento.

Acreditamos que estes métodos deveriam ser reservados para pacientes com função cardíaca estável. Em pacientes que requerem massagem cardíaca, procedimentos diagnósticos deveriam ser reduzidos ao mínimo e o paciente levado ao centro cirúrgico assim que possível.

Em certas situações, diferenciação clínica entre infarto agudo do miocárdio e embolismo pulmonar fulminante pode ser difícil, especialmente quando a história médica, ECG, comprometimento respiratório e raios $X$ de tórax não revelarem sinais típicos. Se a indicação cirúrgica é urgente, a decisão clínica é feita baseada, principalmente, nos resultados do Swan-Ganz (SMIDT et alii ${ }^{17}$ ).

Nós concordamos com GRAY et alii ${ }^{8}$ que decisões em atenção à intervenção cirúrgica têm sido baseadas na necessidade individual do paciente.

Em pacientes com leve ou moderado embolismo pulmonar agudo, a trombólise é a terapia de escoIha, a não ser que haja contra-indicação por outras razões ${ }^{15}$. Contudo, em pacientes requerendo uma dose elevada de catecolaminas e com progressiva hipóxia, necessitando intubação e ventilação mecânica, a terapêutica trombolítica corrente parece inapropriada. Acreditamos que estes pacientes deviam sofrer urgente embolectomia 17.

A experiência tem mostrado que o manuseio não cirúrgico destes pacientes tem sido menos favorável do que aqueles que são submetidos a cirurgia de embolectomia sob contínua ressuscitação. Além do mais, a técnica de embolectomia pulmonar está difundida e descrita por COOLEY et alii ${ }^{3} \mathrm{e}$ SHARP 16.
Em estudo multicêntrico, DEL CAMPO ${ }^{6}$ reviu 651 pacientes operados por esta técnica. A mortalidade média foi de $40 \%$, próxima da obtida por nós, comparada com $51 \%$ para pacientes operados sem CEC.

GRAY et alii ${ }^{9}$ relatam uma mortalidade hospitalar de $11 \%$ em pacientes que não tiveram parada cardíaca sustentada antes da embolectomia e $64 \%$ nos que tiveram.

Há uma diferença de opinião se pacientes requerendo permanente massagem cardíaca externa deveriam ser referidos para intervenção cirúrgica.

Nós advogamos esternotomia mediana rápida, com canulação arterial e venosa durante massagens cardíacas externa e interna intermitentes 17 Outros grupos preferem circulação fêmora-femoral parcial ${ }^{13}$. Acreditamos ser este procedimento reservado a pacientes que não podem imediatamente sofrer a operação, seno beneficiados com a estabilização cardiovascular pré-operatória.

$\mathrm{Na}$ experiência de outros, a canulação dos vasos femorais e CEC instituída ao lado do leito não ganham tempo e podem atrasar a embolectomia ${ }^{17}$

Felizmente, a EPM ocorre, infreqüentemente, após operações cardiacas, possivelmente devido a heparinização sistêmica intra-operatória e uso de antiagregantes no período de pós-operatório ${ }^{7}$.

Em adição, a CEC causa mudanças nos elementos do sangue que retardam a formação do coágulo, mudanças que incluem consumo de fatores da coagulação, ativação fibrinolítica em cascata, trombocitopenia, disfunção plaquetária e hemodiluição ${ }^{1}$.

Somente dois fatores de risco específicos para EPM têm sido descritos: hiperlipidemia e reparo de defeito septal atrial. Sete por cento dos pacientes com hipoplasia tipo II sofrem EPM após operações cardíacas e 1,5\% após reparo de defeito septal atrial ${ }^{10}$. Um estado de hipercoagulação pode ser atribuído a estes pacientes.

Acredita-se que pacientes com trombocitopenia induzida por heparina podem ser mais suscetiveis a episódios de EPM.

\section{CONCLUSÕES}

A sobrevida não só reflete a eficácia do manuseio cirúrgico, mas também depende da morbidade pré-embólica, da qualidade do manuseio pré-operatório cardiopulmonar e das complicações pós-operatórias.

Alguns pacientes com embolismo pulmonar agudo podem ser tratados com terapêutica trombolítica e a embolectomia pulmonar somente seria 
ABDULMASSIH NETO, C.; BARBOSA, M. A. O.; PIEGAS, L. S.; CHACCUR, P.; DINKHUYSEN, J, J.; SALERNO, P. R.; ARNONI, A. S.; PAULISTA, P.P.; SOUZA, L. C. B.; JATENE, A. D. - Tratamento cirúrgico da embolia pulmonar maciça aguda. Rev. Bras. Cir. Cardiovasc.,8(2):125-129, 1993.

considerada nos comprometidos hemodinamicamente e naqueles em que a terapêutica tromboembolítica é contra-inicada e também naqueles em que o tempo requerido para o tratamento terapêutico é inaceitável.
Em síntese, nossa experiência demonstra que reanimação efetiva com manutenção de função cerebral não comprometida é possível em pacientes com embolismo pulmonar fulminante, resultando falência cardiopulmonar.

RBCCV 44205-207

ABULMASSIH NETO, C.; BARBOSA, M. A. O.; PIEGAS, L. S.; CHACCUR, P.; DINKHUYSEN, J. J.; SALERNO, P. R.; ARNONI, A. S.; PAULISTA, P. P.; SOUZA, L. C. B.; JATENE, A. D. - Surgical treatment of acute pulmonary embolism. Rev. Bras. Cir. Cardiovasc., 8(2):125-129, 1993.

ABSTRACT: Between January 1984 and December 1992, 8 patients with acute massive pulmonary embolism (PE) underwent pulmonary embolectomy under cardiopulmonary bypass. The age ranged from 36 to 70 years (average 56.6 years). There were 6 men and 2 women. A causative factor for pulmonary embolism was found in 6 (95\%): myocardium revascularization in 3, abdominal lipoaspiration in 2 and hemorrhoidectomy in 1. All patients where severely hypoxic with mean arterial $\mathrm{PO}_{2}$ of $55 \%$. The diagnosis of $\mathrm{PE}$ was established by pulmonary angiogram in $6(75 \%)$ and surgery in 2 . Hospital mortality was $50 \%$ (4 patients), two of them had previous cardiac arrest, 1 patient died of acute respiratory distress syndrome, 1 of neurologic complication, 1 of pulmonary infection and 1 of myocardial failure. Among the survival patients, 3 are without symptoms and 1 complains of discrete exertional dyspnea (NYHA class II). This study demonstrates that:

1) the mortality rate was higher in patients with previous cardiac arrest;

2) the time between diagnosis and surgery was a predictive factor;

3 ) the high mortality rate reflects the gravity of the situation;

4) the long term results in surviving patients have been favorable.

DESCRIPTORS: pulmonary embolism, surgery.

\section{REFERÊNCIAS BIBLIOGRÁFICAS}

1 BAGGE, L.; LILIENBERG, G.; NYSTRON, S. O. Coagulation fibrinolysis and bleeding after open-heart surgery. Scand. J. Thorac. Cardiovasc. Surg., 20: 151-160, 1986.

2 BEALL Jr., A. C. - Pulmonary embolectomy.

Ann. Thorac. Surg., 51: 179-185, 1991.

3 CLARKE, D. B. - Pulmonary embolism re-evaluated. Ann. R. Coll. Surg. Engl., 63: 18-24, 1981.

4 COOLEY, D. A.; BEALL Jr., A. C.; ALEXANDER, J. K. - Acute massive pulmonary embolism: successful surgical treatment using temporary cardiopulmonary bypass. JAMA, 177: 283-286, 1961.

5 COON, W. W. \& COLLER, F. A. - Some epidemiologic considerations of thromboembolism. Surg. Gynecol. Obstet., 109: 487-501, 1959.

6 DEL CAMPO, C. - Pulmonary embolectomy: a review. Can. J. Surg., 28: 111-113, 1985.

7 GILLINOV, T.; DAVIS, E. A.; ALBERG, A. J.; RYKIEL, M.; GARDNER, T. J.; CAMERON, D. E. - Pulmonary embolism in the cardiac surgical patient. Ann. Thorac. Surg., 53: 988-991, 1992.

8 GRAY, H. H.; MILLER, G. A. H.; PANETH, M. Pulmonary embolectomy: its place in the management of pulmonary embolism. Lancet, 1: 1441-1445, 1988.

9 GRAY, H. H.; MORGAN, J. M.; PANETH, M.; MILLER, G. A. H. - Pulmonary embolectomy: indication and results. Br. Heart. J., 57: 572-579, 1987.

10 HANSON, E. C.; LEVINE, F. H.; ADZICK, N. S. - Early and late results of coronary artery bypass in patients with hyperlipoproteinemia. J. Thorac. Cardiovasc. Surg., 79: 372-380, 1980.

11 KARLSON, J. K. - Discussion in fulminant pulmonary embolism. Ann. Thorac. Surg., 52: 1102-1107, 1991.

12 KIRSCHNER, M. - Ein Durch die trendelenburgsche Operation geheiter Fall embolic der Art. pulmonalis. Arch. Klin. Chir., 133: 312-359, 1924.

13 MATOX, K. L.; FELDTMAN, R. W.; BEOLL, A. C.; DeBAKEY, M. E. - Pulmonary embolectomy for acute 
ABDULMASSIH NETO, C.; BARBOSA, M. A. O.; PIEGAS, L. S.; CHACCUR, P.; DINKHUYSEN, J, J.; SALERNO, P. R.; ARNONI, A. S.; PAULISTA, P.P.; SOUZA, L. C. B.; JATENE, A. D. - Tratamento cirúrgico da embolia pulmonar maciça aguda. Rev. Bras. Cir. Cardiovasc.,8(2):125-129, 1993.

massive pulmonary embolism. Ann. Surg., 6: 726$731,1982$.

SAUTHER, R. D.; MYERS, W. O.; RAY, J. F.; WENZEL, F. J. - Pulmonary embolectomy: review and current status. Progr. Cardiovasc. Dis., 5: 371-389, 1975.

SCHOLZ, K. H.; HILMER, T.; SCHUESTER, S.; KREUZER, H.; TEBLE, U. - Trombolyze bei reanimierten Patient mit Lungenembolic. Dtsch. Med. Wochenschr., 115: 930-935, 1990.

SHARP, E. H.; - Pulmonary embolectomy: successful removal of massive pulmonary embolism with the support of cardiopulmonary bypass: case report. Ann. Surg., 156: 1-4, 1926.

17 SHMIDT, C.; ZIETLOW, S.; WAGNER, T. O. F.; LAAS J.; BORST, H. - Fulminant pulmonary embolism: symptoms, diagnostics, operative technique and results. Ann. Thorac. Surg., 52: 1102-1107, 1991.

TRENDELENBURG, F. - Ueber dir operative Behandlung der Embolic der Lungenraterie. Arch. Klin. Chir. 86: 201-232, 1908.

TSCHIRKOV, A.; KRAUSE, E.; ELERT, O.; SATTER, P. - Surgical management of massive pulmonary embolism. J. Thorac. Cardiovasc. Surg., 75: 730-733, 1977.

\section{Discussão}

\section{DR. FÁBIO JATENE}

São Paulo, SP

Eu gostaria, a título de contribuição ao trabalho apresentado pelo Dr. Camilo, de projetar imagens de um filme no momento da retirada dos trombos do iterior da árvore arterial pulmonar. Como se pode observar, os trombos de côr vinhosa escura e que caracterizam uma embolia maciça aguda encontram-se no tronco da artéria pulmonar, bem como os seus ramos direito e esquerdo. A retirada à esquerda é feita por abertura do tronco da artéria pulmonar com prolongamento para o ramo esquerdo e à direita, por outra abertura separada, no ramo direito da artéria pulmonar. Pode-se observar que não há uma firme aderência dos tronbos à parede da artéria e a sua retirada pode ser realizada por suave contra-tração e dissecção romba. Neste caso, deve-se atentar para possiveis fragmentos que permaneçam nas posições distais de artéria pulmonar. A visão do trombo retirado já mostra um princípio de organização do mesmo com os seus prolongamentos para os ramos arteriais lobares e segmentares. Entretanto, quando os casos são mais crônicos e já há uma parcial ou completa organização dos trombos, deve-se atentar ao fato de que, nestes casos, os trombos, de aspecto diverso, mais organizados e de côr clara, devem ser retirados por contra - tração mais vigorosa e ressecção tipo tromboendarterectomia, com retirada conjunta do trombo e da endartéria. Nós temos uma experiêcia acumulada de 12 pacientes, nos quais foi realizada a tromboendarterectomia crônica, com $92 \%$ deles recebendo alta hospitalar e $79 \%$ de bons resultados funcionais. Com relação aos casos agudos, tenho algumas colocações ao Dr. Camilo. É sabido que, já há vários anos, como comprovam os trabalhos cooperativos de 1974, publicados no "JAMA", e os estudos de 1977, de Miller, publicados no "American Heart Journal", que se advoga o uso de trombolíticos mesmo nos casos de tromboembolia maciça aguda. Dentre as contra-indicações, estaria a presença de cirurgias prévias, mas é de nosso conhecimento que vários Serviços, mesmo entre nós, têm utilizado a trombólise nestes casos, sem maiores problemas. Eu gostaria de saber se ela foi usada em alguns de seus casos (havia 2 casos sem cirurgia prévia) e, se não, por que? Outro ponto é que os vários métodos de assistência circulatória, hoje em dia, podem ser realizados em boa parte dos nossos Serviços. O acesso artério-venoso fêmoro-femoral preconizado por alguns autores pode ser facilmente realizado por punção, em questão de minutos, mesmo em UTI, e proporciona maior tempo e melhores condições para o paciente ser encaminhado à cirurgia. Como o Sr. vê esta abordagem? Gostaria, por fim, de cumprimentá-lo pelo trabalho.

\section{DR. ABDULMASSIH NETO (Encerrando)}

Agradeço os comentários do Dr. Fábio. Quero, entretanto, ressaltar que o filme ora apresentado é de outra entidade clínica, totalmente diferente da nossa série de pacientes, que são agudos. Respondendo às suas questões, podemos dizer que as decisões sobre a indicação terapêutica clínica ou cirúrgica deve ser baseada na necessidade indivídual do paciente. Em pacientes com leve ou moderado embolismo pulmonar agudo, a trombólise é a terapia de escolha, a menos que haja contra-indicação por outras razões. Os nossos pacientes apresentavam embolia pulmonar maciça aguda, com mais de $75 \%$ da árvore pulmonar comprometida e grave hipóxia, o que inviabiliza, a nosso ver, o uso de trombolíticos e, portanto, não foi administrado neste grupo. A última questão é pertinente, pois a conduta moderna na embolia pulmonar maciça, com grave repercussão hemodinâmica, com ou sem parada cardiocirculatória, além das manobras de ressuscitação cardíaca, inclui a abordagem dos vasos femorais com equipamento de CEC portátil, com pessoal médico e paramédico bem treinados. 